\title{
Reproducibility of CMR right ventricle volumetric measurements is independent of reader experience under a standardized protocol
}

\author{
Murilo Foppa ${ }^{1 *}$, Arman Ashrafi ${ }^{1}$, Carol J Salton ${ }^{1}$, Michael L Chuang ${ }^{2}$, Warren J Manning ${ }^{1}$ \\ From 17th Annual SCMR Scientific Sessions \\ New Orleans, LA, USA. 16-19 January 2014
}

\section{Background}

The right ventricle's complex geometry makes it challenging to measure its size and function. Though volumetric CMR is generally considered the gold standard, standardized reading protocols are necessary. We sought to investigate whether a specific reading protocol can supersede cardiology training in right ventricular (RV) measurements. We aimed to evaluate the reproducibility of RV measurements done by both an experienced and a novice CMR reader.

\section{Methods}

During a training period an experienced (Level III trained) CMR reader and a CMR novice (high school student) jointly interpreted 50 cine SSFP studies obtained in the left ventricular short-axis orientation as contiguous stack encompassing both ventricles in their entirety. The standardized endocardial tracing protocol was delimited by the pulmonary valve and the tricuspid valve planes; interpretation was augmented using cinereview and cross-referencing with orthogonal cine images.
RV stroke volume (RVSV) and ejection fraction (RVEF) were calculated from end diastolic volume (RVEDV) and end systolic volume (RVESV). After the training period, we randomly selected 24 anonymized datasets from a representative sample $(50 \%$ men; median age 63 years[range 48-78]), and the two readers independently read the studies twice, in a random order and on different days. Reproducibility was evaluated with coefficients of variation $(\mathrm{CV})$ and concordance correlation coefficients (Rho).

\section{Results}

Mean \pm SD measurements and CV did not differ between the experienced and the novice reader, respectively, for RVEDV $(124.4 \pm 30.1$ vs $125.0 \pm 30.1 \mathrm{~mL}$; CV: 0.05 vs $0.04 ; \mathrm{P}=\mathrm{NS})$, RVESV (45.6 $\pm 14.445 .8 \pm$ $13.4 \mathrm{~mL}$; CV: 0.11 vs $0.09 ; \mathrm{P}=\mathrm{NS})$, RVSV $(78.9 \pm 18.8$ vs $79.2 \pm 18.7 \mathrm{~mL} ; \mathrm{CV}: 0.05$ vs $0.03 ; \mathrm{P}=\mathrm{NS})$, RVEF $(63.6 \pm 5.4$ vs $63.4 \pm 5 \%$; CV: 0.04 vs $0.04: \mathrm{P}=\mathrm{NS})$. The concordance correlation coefficients and 95\% limits of agreement are shown in the Table 1.

Table 1 Intrarreader and interreader concordance correlation coefficients (Rho) and mean differences with $95 \%$ limits of agreement

\begin{tabular}{ccccccc}
\hline & \multicolumn{2}{c}{ Experienced Reader } & \multicolumn{2}{c}{ Novice reader } & \multicolumn{2}{c}{ Interreader } \\
\hline & Rho(95\%Cl) & Diff $(\mathbf{9 5} \% \mathbf{C l})$ & Rho(95\%Cl) & Diff $(\mathbf{9 5 \% C l )}$ & Rho(95\%Cl) & Diff (95\%Cl) \\
\hline RVEDV & $0.98(0.96-1.0)$ & $1.1(-11,13)$ & $0.99(0.98-1.0)$ & $1.35(-8,10)$ & $0.94(0.90-0.98)$ & $0.1(-9,9)$ \\
\hline RVESV & $0.91(0.85-0.97)$ & $3(-7,13)$ & $0.96(0.92-0.98)$ & $1.8(-6,10)$ & $0.93(0.89-0.98)$ & $0.1(-9,9)$ \\
\hline RVSV & $0.97(0.94-0.99)$ & $-1.8(-11,7)$ & $0.99(0.99-1.0)$ & $-0.5(-5,4)$ & $0.97(0.96-1.0)$ & $-0.7(-8,7)$ \\
\hline RVEF & $0.81(0.68-0.95)$ & $-1.8(-7,4)$ & $0.92(0.88-0.98)$ & $-0.9(-5,3)$ & $0.89(0.80-0.98)$ & $-0.2(-5,4)$ \\
\hline
\end{tabular}

${ }^{1}$ Cardiology, Beth Israel Deaconess Medical Center, Boston, Massachusetts,

USA

Full list of author information is available at the end of the article 


\section{Conclusions}

All global volumetric CMR RV measurements were highly reproducible, suggesting that a standardized reading protocol overcomes the complex RV geometry. Background CMR level of training did not affect these results. The high reproducibility and feasibility of applying a specific training protocol may impact research, allowing smaller sample sizes and better logistics for reading as well as for serial clinical assessment.

\section{Funding}

Dr Murilo Foppa has received support from CAPESBrazil (9601-11-2).

\section{Authors' details}

${ }^{1}$ Cardiology, Beth Israel Deaconess Medical Center, Boston, Massachusetts, USA. ${ }^{2} \mathrm{NHLBI}$ 's Framingham Heart Study, Framingham, Massachusetts, USA.

Published: 16 January 2014

- Convenient online submission

- Thorough peer review

- No space constraints or color figure charges

- Immediate publication on acceptance

- Inclusion in PubMed, CAS, Scopus and Google Scholar

- Research which is freely available for redistribution 